\title{
Influencia de la agitación intensa del efluente en diálisis peritoneal automática para el cálculo de la dosis de diálisis
}

\author{
Dolores Marquina Parra \\ Conchita Blasco Cabañas \\ Nuria Mañé Buixo \\ Esther Ponz Clemente \\ Vicente Esteve Simó \\ Carmen Grau Pueyo \\ Juan Carlos Martínez Ocaña \\ Elios Yuste Jiménez \\ Manuel García García
}

Corporación Parc Taulí Sabadell (Barcelona)

\section{RESUMEN}

Introducción. La metodología, toma de muestras de sangre y del efluente peritoneal (EP) y fórmulas utilizadas para la realización de cálculos de la dosis de diálisis peritoneal debe ser estándar. Las guías DOQI recomiendan la mezcla de todo el EP y su agitación intensa.

Objetivo. Estudiar si había diferencias en la determinación de Cry de urea antes y después de agitar el EP y si afectaban al cálculo de $\mathrm{KT} / \mathrm{Vs}$ y del $\mathrm{ClCr} / 1.73 \mathrm{~m}^{2}$.

Material y métodos. En 13 enfermos estables en DPA se realizaron 25 determinaciones de urea y $\mathrm{Cr}$ antes y después de agitar la garrafa del EP. Se calculó el KT/Vs y el ClCr/1.73 m2 de la DP con la muestra de sangre obtenida 1-3 horas después de la finalización del tratamiento.

Correspondencia:

Dolores Marquina

Servicio de Nefrología

Corporació Parc Taulí s/n

08207 Sabadell
Conclusiones. La agitación del EP previa a la toma muestras no modificó el resultado de urea y $\mathrm{Cr}$ y por consiguiente del $\mathrm{KT} / \mathrm{Vs}$ y $\mathrm{ClCr} / 1.73 \mathrm{~m} 2$ en nuestros enfermos. Los diferentes resultados con otros estudios pueden explicarse por otros factores como el tiempo transcurrido desde el final de la DPA y la recogida la muestra o el volumen total.

\section{PALABRAS CLAVE: AGITACIÓN EFLUENTE PERITONEAL CÁLCULO DOSIS DIÁLISIS}

\section{INFLUENCE OF INTENSE SHAKING OF THE EFFLUENT IN AUTOMATIC PERITONEAL DIALY- SIS IN CALCULATING THE DIALYSIS DOSAGE}

\section{ABSTRACT}

Introduction The methodology, taking of blood and peritoneal effluent (PE) samples and formulae used to calculate the dosage of peritoneal dialysis should be standard. DOQI guidelines recommend mixing all the $\mathrm{PE}$ and shaking it well.

Objective. To study whether there were differences in determining $\mathrm{Cr}$ and urea before and after shaking the PE and whether they affected the calculation of $\mathrm{KT} / \mathrm{Vs}$ and $\mathrm{ClCr} / 1.73 \mathrm{~m}^{2}$. 
Material and methods. In 13 stable patients on APD 25 urea and $\mathrm{Cr}$ tests were carried out before and after shaking the $\mathrm{PE}$ container. The $\mathrm{KT} / \mathrm{Vs}$ and $\mathrm{ClCr} / 1.73$ $\mathrm{m}^{2}$ of the $\mathrm{P}^{\wedge} \mathrm{D}$ were calculated with the blood sample obtained 1-3 hours after finishing treatment.

Conclusions. Shaking the PE prior to taking samples did not modify the result in urea and $\mathrm{Cr}$ and therefore of the $\mathrm{KT} / \mathrm{Vs}$ and $\mathrm{ClCr} / 1.73 \mathrm{~m}^{2}$ in our patients. The different results compared to other studies may be explained by other factors such as the time elapsed since the end of the APD and the taking of the sample or the total volume.

\section{KEY WORDS:}

\section{SHAKING PERITONEAL EFFLUENT} CALCULATION OF DIALYSIS DOSAGE

\section{INTRODUCCIÓN}

En el control y seguimiento de un enfermo en Diálisis Peritoneal (DP), junto con la valoración clínica global se recomienda la obtención de unos índices de adecuación dialítica: el KT/V de urea (KT/V) y el Aclaramiento de Creatinina por $1.73 \mathrm{~m}^{2}(\mathrm{ClCr})$ semanales ${ }^{(1)}$. Todas las guías clínicas desarrolladas por distintas instituciones y sociedades científicas aconsejan unos niveles determinados de $\mathrm{KT} / \mathrm{V}$ y $\mathrm{ClCr}$ calculados con el líquido peritoneal y la orina de 24 horas $^{(1-4)}$. Para el seguimiento clínico es importante el cálculo del KT/N y ClCr totales, es decir del aportado por la DP y la función renal residual. La metodología, toma de muestras de sangre y del efluente peritoneal y las fórmulas utilizadas deberían ser estándares. En el caso de la Diálisis Peritoneal Automática con Cicladora (DPA), la recomendación de las guías DOQI americanas es que la muestra de sangre se tome en la mitad de la permanencia larga y que el efluente peritoneal se agite intensamente. Existe poca información sobre la metodología práctica de la obtención y manipulación de las muestras de líquido peritoneal de 24 horas, sobre todo en relación al cálculo del volumen total del efluente $e^{(5)}$. En el caso de la DPA existen varias opciones para calcular el volumen total de líquido peritoneal; contabilizar en una probeta calibrada todo el líquido, pesar el contenedor y restar la tara o considerar el líquido drenado a través de la información obtenida con la cicladora. Otro tema a considerar en la obtención de la muestra del líquido de 24 horas es la recomendación de que se mezcle bien todo el líquido. En el concepto de mezclar se incluye el vaciado de todo el contenido de las bolsas de drenaje de líquido peritoneal en el mismo contenedor y de removerlo bien antes de tomar la muestra definitiva. En un estudio realizado en DPA con muestras del líquido peritoneal antes y después de agitar el contenido observaban que los resultados en la muestra sin agitar infravaloraban las concentraciones, aunque únicamente en el caso de la creatinina había diferencias significativas ${ }^{(6)}$. En este estudio el contenedor era una bolsa de drenaje y los enfermos eran adiestrados para invertir la bolsa 10 veces. En nuestro caso todos los enfermos en DPA utilizan un contenedor de plástico (garrafa) para la obtención de la muestra de 24 horas de líquido peritoneal. Los enfermos toman la muestra directamente de la garrafa, pero nunca se les había advertido que mezclaran bien, o agitaran, el contenido de la garrafa antes de tomar la muestra.

El objetivo de nuestro estudio es analizar si hay diferencias en la determinación de urea y creatinina antes y después de agitar intensamente el efluente peritoneal y si afecta al cálculo del KT/N y el ClCr.

\section{MATERIAL Y MÉTODOS}

Estudio prospectivo realizado en una Unidad de Diálisis Peritoneal en enfermos en DPA. Se solicitó consentimiento informado verbal a los enfermos para su colaboración en el estudio. Todos los enfermos realizan DPA con la cicladora Home-Choice Pro de Baxter. Los enfermos se encontraban en situación de estabilidad clínica y ninguno había sufrido un episodio de peritonitis en lo últimos 3 meses.

A los enfermos se les instruía para la obtención de dos muestras del efluente peritoneal. Una primera muestra del contenido de la garrafa antes (Muestra A: sin mezcla) y después de agitar bien (Muestra B: con mezcla) todo el contenido con cualquier utensilio alargado.

El cálculo del volumen del efluente se obtuvo mediante la suma del volumen infundido, el drenaje inicial, la ultrafiltración total nocturna, las ultrafiltraciones diurnas y el volumen de cebado y de vaciado de bolsas a la garrafa que no pasaban por el peritoneo.

Las muestras de sangre se han obtenido siempre entre 1-2 horas después de finalizar el tratamiento con la ci- 
cladora. Las determinaciones de urea y creatinina se realizaron en un autoanalizador mediante las técnicas de ureasa ultravioleta y de jaffé cinético, respectivamente.

Se calculó el KT/N y el CLCr/1.73 m2 semanal peritoneal y total (incluyendo la función renal residual), utilizando el paquete estadístico de PD-ADEQUEST ${ }^{\circledR}$ 2.0. Se transformó la urea en nitrógeno ureico en sangre (BUN). Los cálculos se han realizado utilizando el paquete estadístico SPSS para Windows versión 11.5. Se han expresando los resultados como media y desviación estándar y se ha realizado una estadística básica descriptiva, analizando las diferencias de cada dato apareado.

\section{RESULTADOS}

Se han realizado un total de 25 estudios en 13 pacientes, 8 varones, con una edad media de 46.58 años, desde 21 a 75 años, y un tiempo medio de permanencia en DP de 26.62 meses, desde 6 a 72 meses. De los 13 enfermos, 4 realizaban DPA con día seco y otros 4 enfermos realizaban 1 o 2 intercambios diurnos. Seis enfermos no tenían función renal residual. Tres enfermos en DPA no se incluyeron en el estudio por problemas de inestabilidad clínica.

En la tabla 1 se expresan los resultados de urea y creatinina en las muestras A y $\mathrm{B}$, antes y después de agitar el contenido de la garrafa del efluente peritoneal. En 15 casos la creatinina fue igual, en 7 casos fue inferior y en 3 superior antes que después de remover. Para la urea encontramos que en 6 casos no se modificó, en 5 casos fue superior y en 14 inferior antes de remover.

\begin{tabular}{|l|lll|}
\hline & $\begin{array}{l}\text { Muestra A } \\
\text { Sin mezcla }\end{array}$ & $\begin{array}{l}\text { Muestra B } \\
\text { Con mezcla }\end{array}$ & $\begin{array}{l}\text { Media de las } \\
\text { diferencias }\end{array}$ \\
\hline Creatinina LP & $3.79 \pm 1.30 \mathrm{mg} / \mathrm{dL}$ & $3.74 \pm 1.36 \mathrm{mg} / \mathrm{dL}$ & $-0.04 \pm 0.18$ \\
\hline BUN LP & $37.91 \pm 10.13 \mathrm{mg} / \mathrm{dL}$ & $37.53 \pm 9.88 \mathrm{mg} / \mathrm{dL}$ & $-0.38 \pm 2.72$ \\
\hline
\end{tabular}

Tabla 1. Valores de Creatinina y Urea en el efluente peritoneal antes y después de agitar la garrafa.

En general no se observaron diferencias apreciables entre las dos muestras, tanto para la creatinina como para la urea. En ningún caso la diferencia observada entre los dos resultados superaba al coeficiente de variabilidad de la técnica de determinación para la creatinina y la urea de nuestro laboratorio. Al obtener resultados a simple vista tan similares no hemos realizado ninguna prueba estadística.

En la tabla 2 se expresan los resultados obtenidos para el cálculo de la dosis de diálisis con las dos muestras, considerando sólo lo aportado por la Diálisis Peritoneal.

\begin{tabular}{|l|lll|}
\hline & $\begin{array}{l}\text { Muestra A } \\
\text { Sin mezcla }\end{array}$ & $\begin{array}{l}\text { Muestra B } \\
\text { Con mezcla }\end{array}$ & $\begin{array}{l}\text { Media de las } \\
\text { diferencias }\end{array}$ \\
\hline $\mathrm{KT} / \mathrm{Ns} \mathrm{DP}$ & $1.67 \pm 0.58$ & $1.69 \pm 0.60$ & $0.014 \pm 0.11$ \\
\hline $\mathrm{ClCr} / 1.73 \mathrm{~m}^{2} \mathrm{DP}$ & $41.01 \pm 18.63$ & $40.54 \pm 19.10$ & $-0.46 \pm 1.84$ \\
\hline
\end{tabular}

Tabla 2. Valores de $\mathrm{KT} / \mathrm{Vs}$ y $\mathrm{ClCr} / 1.73 \mathrm{~m}^{2}$ obtenidos por la Diálisis Peritoneal.

En la tabla 3 se detallan los resultados obtenidos para el $\mathrm{KT} / \mathrm{Vs}$ y el $\mathrm{ClCr} / 1.73 \mathrm{~m}^{2}$, considerando la Diálisis $\mathrm{Pe}$ ritoneal y la Función Renal Residual.

\begin{tabular}{|llll} 
& $\begin{array}{l}\text { Muestra A } \\
\text { Sin mezcla }\end{array}$ & $\begin{array}{l}\text { Muestra B } \\
\text { Con mezcla }\end{array}$ & $\begin{array}{l}\text { Media de las } \\
\text { diferencias }\end{array}$ \\
\hline $\mathrm{KT} / \mathrm{Ns}$ Total (DP/FRR) & $2.15 \pm 0.42$ & $2.17 \pm 9.42$ & $0.015 \pm 0.11$ \\
\hline $\mathrm{ClCr} / 1.73 \mathrm{~m}^{2}$ Total (DP/FRR) & $65.24 \pm 18.01$ & $64.91 \pm 18.21$ & $-0.25 \pm 1.20$ \\
\hline
\end{tabular}

Tabla 3. Valores de $\mathrm{KT} / \mathrm{Vs}$ y $\mathrm{ClCr} / 1.73 \mathrm{~m}^{2}$ aportados por la suma de la Diálisis Peritoneal y la función renal residual.

Como es lógico, al no haber variaciones apreciables en las cifras de urea y creatinina del dializado antes y después de remover intensamente el líquido de la garrafa, las diferencias en los resultados del KT/Vs y el $\mathrm{ClCr} / 1.73 \mathrm{~m}^{2}$ no tienen relevancia clínica.

\section{DISCUSIÓN}

No hemos observado que la agitación intensa del efluente peritoneal de la garrafa de drenaje modifique en un sentido $\mathrm{u}$ otro la determinación de urea y creatinina y consiguientemente el cálculo de la dosis de diálisis. Nuestros resultados difieren de los obtenidos en el estudio de Kannapadi(6) en el que observaron un aumento de la concentración de urea y creatinina después de agitar la bolsa de drenaje, alcanzando significación estadística en el caso de la creatinina. $\mathrm{El} \mathrm{ClCr} / 1.73 \mathrm{~m}^{2}$ pasaba de $50.66 \pm 12,1$ a $54.5 \pm 11.3$ con un aumento del $7 \%$. En nuestro caso el $\mathrm{ClCr} / 1.73 \mathrm{~m}^{2}$ de la Diálisis Perito- 
neal pasaba de $41.01 \pm 18.63$ a $40.54 \pm 19.10$. La diferencia es inapreciable e incluso tiene una tendencia a disminuir en la muestra después de removerla. Nosotros esperábamos obtener un aumento de los niveles de urea y creatinina después de remover bien todo el contenido de la garrafa porque la recomendación general es que cualquier líquido biológico debe ser agitado antes de tomar una muestra para obtener un resultado fiable.

Todas las guías clínicas recomiendan tomar la muestra después de mezclar y agitar el efluente y parece lógico que pueda tener influencia. Es frecuente que se manejen hasta 15 o 20 litros de líquido y dependiendo del tiempo transcurrido desde la finalización de la DPA hasta la toma de la muestra, el líquido podría sedimentarse y una muestra obtenida de la superficie infravalorar incluso el resultado de moléculas de pequeño tamaño como la urea y la creatinina. Cualquier líquido tiene un movimiento propio que hace que su tendencia sea a mezclarse de manera uniforme, pero en los líquidos biológicos existen restos celulares y proteicos (fibrina) que puede formar sedimentos por gravedad y modificar la concentración de las sustancias, probablemente más en el caso de moléculas de mayor tamaño como las proteínas. En realidad los resultados iniciales de los valores brutos de urea y creatinina en las muestras de antes y después de agitar el líquido peritoneal no superan las posibles variaciones debidas a la propia técnica del laboratorio. Así el coeficiente de variabilidad para el estándar alto de la creatinina (Creatinina de $5.09 \mathrm{mg} / \mathrm{dL}$ ) es de 2.93 con una desviación estándar de 0.15 , por lo que se puede considerar que para la cifra de creatinina de 5.09 $\mathrm{mg} / \mathrm{dL}$, podríamos encontrar variaciones desde 4.79 a $5.39 \mathrm{mg} / \mathrm{dL}$, con un intervalo de confianza del 95 \%. Ésta variabilidad podría considerarse aceptable para las normas de calidad y calibración de las técnicas de laboratorio. Como podemos observar las variaciones de nuestros resultados son inferiores a la propia variabilidad del método. Lo mismo pasa con el coeficiente de variabilidad de la urea para estándar alto (104 mg/dL) y el bajo (32 mg/dL).

Ante la falta de diferencias y la contradicción con los resultados del estudio de Kannapadi nos hemos planteado la posibilidad de errores en el etiquetado de las muestras, pero en cualquier caso, las diferencias entre la cifra de urea y creatinina en las muestras pareadas son muy bajas. Nosotros hemos estudiado 25 muestras pareadas y Kannapadi estudió sólo 11 y no reflejan los datos de la cifra absoluta de urea y creatinina de las muestras ni la posibilidad de que las variaciones pudieran ser explicadas por el coeficiente de variabilidad de su laboratorio. Otra posible explicación es que el tiempo transcurrido desde el final de la terapia hasta la toma de la muestra influya de manera determinante en el resultado, es decir que si la muestra se recoge inmediatamente no hayan cambios y si se esperan varias horas sí. En el caso de que se recoja inmediatamente, tenemos un contenedor, en nuestro caso una garrafa, a la que se aboca una línea y va cayendo líquido peritoneal, con lo que se está produciendo una agitación provocada por la caída continua de líquido. En el estudio de Kannapadi no se utilizaba una garrafa, sino que era una bolsa contenedor con capacidad para 20 litros y se fijaba el tiempo transcurrido desde el final de la DPA hasta la toma de muestras en menos de dos horas. En nuestro caso el tiempo transcurrido era también inferior a dos horas, pero podía ser variable desde 5 minutos a dos horas y ésta variable no se ha recogido. Tampoco podemos descartar que tenga influencia el volumen total de líquido peritoneal que puede oscilar entre 10 y más de 20 litros. En cualquier caso en nuestra experiencia podemos concluir que agitar el efluente peritoneal antes de la toma de muestras no tiene influencia en los resultados del cálculo de la dosis de diálisis y que no es necesario recomendar a los enfermos que remuevan el contenedor.

\section{CONCLUSIONES}

Mezclar y agitar intensamente el efluente obtenido en Diálisis Peritoneal Automática con Cicladora no modifica el resultado de la concentración de urea y creatinina y por consiguiente el cálculo de la dosis de diálisis. En vistas a este resultado no modificaremos nuestra recomendación de tomar una muestra de todo el efluente peritoneal mezclado pero sin realizar una agitación del contenedor.

\section{BIBLIOGRAFÍA}

1. National Kidney Foundation. K/DOQI clinical practice guidelines for peritoneal dialysis adequacy, 2000. Am J Kidney Dis 2000; 37 (Suple 1): S65-136.

2. Blake PG, Bargman JM, Bick J, et al. Guidelines for adequacy and nutrition in peritoneal dialysis. $\mathrm{J}$ Am Soc Nephrol 1999; 10: S287-S321.

3. Gutiérrez Camacho M, Lorite Godoy M, García Gonzalez AI, Hilara Rico L. Recogida de muestras para 
KT/V en diálisis peritoneal continúa automática (DPA). En: Manual práctico de diálisis peritoneal. Editores: Coronel F, Montenegro J, Segas R, Celadilla O, Tejuca M. Atrium Comunicación Estratégica S.L., Badalona 2005.

4. Guías CARI.

http://www.aatrm.net/html/ca/dir214/doc7820.h tml.
Australian and New Zealand Society of Nephrology

5. Bernardini J, Florio T, Bender F, Fried L, Piraino B. Methods to determine drain volume for peritoneal dialysis clearances. Perit Dial Int 2004; 24:182-5.

6. Kannapadi U, Bernardini J. Piraino B. Is small molecules clearance dependent on timing of serum samples and mixing of effluent in CCPD patients? Perit Dial Int 1999; 19: 391-393. 